\title{
STATUS AND PERSPECTIVES OF MINI-MEGATORTORA WIDE-FIELD MONITORING SYSTEM WITH HIGH TEMPORAL RESOLUTION
}

\author{
S. Karpov ${ }^{1}$, G. Beskin ${ }^{1}$, S. Bondar ${ }^{2}$, A. Perkov ${ }^{2}$, E. Ivanov $^{2}$, \\ A. Guarnieri ${ }^{3}$, C. Bartolini ${ }^{3}$, G. Greco ${ }^{4}$, A. Shearer ${ }^{5}$ and V. Sasyuk ${ }^{6}$
}

\begin{abstract}
Here we briefly summarize our long period experience of constructing and operating wide-field monitoring cameras with subsecond temporal resolution to look for optical components of GRBs, fast-moving satellites and meteors. General requirements for hardware for such systems are discussed along with algorithms of real-time detection and classification of various kinds of short optical transients. We also give a status report on the next generation, multi-objective and transforming monitoring system, the MegaTORTORA, whose 6channel (Mini-MegaTORTORA-Spain) and 9-channel prototypes (MiniMegaTORTORA-Kazan) we are building now at SAO RAS. This system combines a wide field of view with subsecond temporal resolution in monitoring regime, and is able to reconfigure itself, in a fractions of second, to follow-up mode which has better sensitivity and provides us with multi-color and polarimetric information on detected transients simultaneously.
\end{abstract}

\section{Introduction}

The systematic study of night sky variability on subsecond time scales still remains an important, but practically unsolved problem. Its necessity for the search of non-stationary objects with unknown localization has been noted by Bondi (1970). Such studies have been performed (Schaefer 1985, 1987), but due to technical limitations it has only been possible either to reach high temporal resolution

\footnotetext{
1 Special Astrophysical Observatory of Russian Academy of Sciences, Russia

2 Institute for Precise Instrumentation, Russia

3 Bologna University, Italy

4 Astronomical Observatory of Bologna, INAF, Italy

5 National University of Ireland, Galway, Ireland

${ }^{6}$ Kazan Federal University, Kazan, Russia
} 
of tens of milliseconds in monitoring of $5^{\prime}-10^{\prime}$ fields, or use $5-10$ seconds time resolution in wider fields. The wide-field monitoring systems currently in operation, such as WIDGET, RAPTOR, BOOTES and $\pi$ of the Sky, while having good sky coverage and limiting magnitude, lack temporal resolution, which significantly lowers their performance in the study of transient events of subsecond duration (as the detection limit degrades as a ratio of event duration to exposure time) or highly variable on subsecond time scales.

In (Karpov et al. 2005; Zolotukhin et al. 2004) we demonstrated that it is possible to achieve the subsecond temporal resolution in a reasonably wide field with small telescopes equipped with fast CCDs, to perform fully automatic searching and classification of fast optical transients. Moreover, a two-telescope scheme Beskin et al. (2005); Karpov et al. (2004), able to study such transients in a very short time after detection, has been proposed. According to these ideas, we created the prototype fast wide-field camera called FAVOR (Karpov et al. 2005) and the TORTORA camera as part of the TORTOREM (Molinari et al. 2006) two-telescope complex, and operated them over several years.

The discovery of the brightest ever GRB, GRB 080319B (the Naked-Eye Burst, Racusin et al. 2008), by several wide-field monitoring systems - TORTORA, RAPTOR and Pi of the Sky - and the subsequent discovery of its fast optical variability on time scales from several seconds down to a sub-second time scale (Beskin et al. 2010b) demonstrated that the ideas behind our efforts in fast temporal resolution wide-field monitoring are correct.

\section{MegaTORTORA - multi-objective transforming instrument}

The parameters defining the field of view size, detection limit and temporal resolution, are mutually exclusive, and are limited by the difficulties of constructing and using objectives with large relative apertures $(D / F \sim 1$ or greater). The only possible way to further improve them simultaneously is to design a multi-objective monitoring system, where detection limit is being improved by decreasing the angular pixel size (Beskin et al. 2007), and field of view - by pointing several identical channels towards different regions of the sky. To operate in a sky background dominated regime, the CCD read-out noise may be suppressed by a high quantum efficiency image intensifier, or by using low-noise EM-CCD or sCMOS as a detector.

Multi-objective design also gives a freedom in the regimes of operation, as fields of view of channels may be either separated or combined, either with the same photometric (or even polarimetric) filter or with combination of different ones.

The MegaTORTORA project Beskin et al. (2010a) we develop according to these lines utilizes the modular design and consists of a set of basic units, 9 objectives each, installed on a separate mounts. Each objective in a unit is placed inside the gimbal suspension with remotely-controlled micro-motors, and so may be oriented independently from others. Also, each objective possesses the set of color and polarization filters, which may be installed before the objective on the fly. It allows to change modes of observation on the fly, from routine wide-field 
monitoring in the color band providing best signal-to-noise ratio (or in a white light, with no filters installed), to the narrow-field follow-up regime, when all objectives are pointed towards the same point, i.e. newly-discovered transient, and observe it in different colors and for different polarization plane orientations simultaneously, to acquire all possible kinds of information for the transient. Simultaneous observation of the transient by all objectives in white light is also possible to get better photometric accuracy by co-adding frames.

Each objective is equipped with the fast EM-CCD, which has a low readout noise even for a high frame rates when the internal amplification is in effect. The data from each channel of such a system, which is roughly 20 megabytes per second, is collected by a dedicated rackmount PC, which stores it in its hard-drive as well as performs its real-time data processing in a way similar to the current processing pipeline of FAVOR and TORTORA cameras, which currently operate under similar data flow rate. The whole system is coordinated by the central server which acquires the transient data from data-processing PCs and controls the pointing and mode of operation of all objectives in response to them.

\section{Mini-MegaTORTORA as a MegaTORTORA prototype}

As a limited realization of a MegaTORTORA concept we designed the prototype design - the Mini-MegaTORTORA, or MMT, which is basically a model of a $3 \times 3$ unit. Main design choice was to use the celostate in a gimbal suspension for a fast repointing of each channel. Such a decision allows to significantly loose the requirements for the structural, dynamical and precision parameters. We are building two variants of Mini-MegaTORTORA with different detectors (image intensifier with fast CCD for MMT-Spain and low-noise sCMOS for MMT-Kazan) and, therefore, slightly different parameters.

Both variants use the CANON EF85 F/1.2 lens as a main objective and celostate mirrors for a fast (faster than $0.3 \mathrm{~s}$ ) repointing in the $\pm 20^{\circ}$ region of the sky. Optical design of a first variant is analogous to the one used in FAVOR (Karpov et al. 2005) and TORTORA (Molinari et al. 2006) systems but with the non-scaling image intensifier. For the second one, it is a bit simple and lacks the image intensifier and transmission optics.

Detector of the first variant is based on a fast Sony IX285AL CCD chip with $6.4 \mu \mathrm{m}$ pixel and $0.13 \mathrm{~s}$ exposure in a continuous acquisition regime, which gives $7.51392 \times 1036$ frames per second with 12 -bit depth. Non-scaling image intensifier has a quantum efficiency of about $25 \%$, and amplified image from its output window is transferred to the CCD by a transmission optics which downscales it 1.7 times; resulting pixel scale is $25^{\prime \prime}$ per pixel and total field of view of a channel is about 100 square degrees. The high image intensifier amplification (of $\sim 150$ ) overcomes CCD read-out noise, but induces its own, spatially-correlated and highly non-poissonian, shot-noise due to ions hitting the photocathode events. Resulting limiting magnitude in differential imaging mode is about $B \sim 12^{\mathrm{m}}$; it is somewhat worse in direct imaging regime due to spatial correlation of the dominant image intensifier noise. Also, direct imaging suffers from the non-uniform spatial 


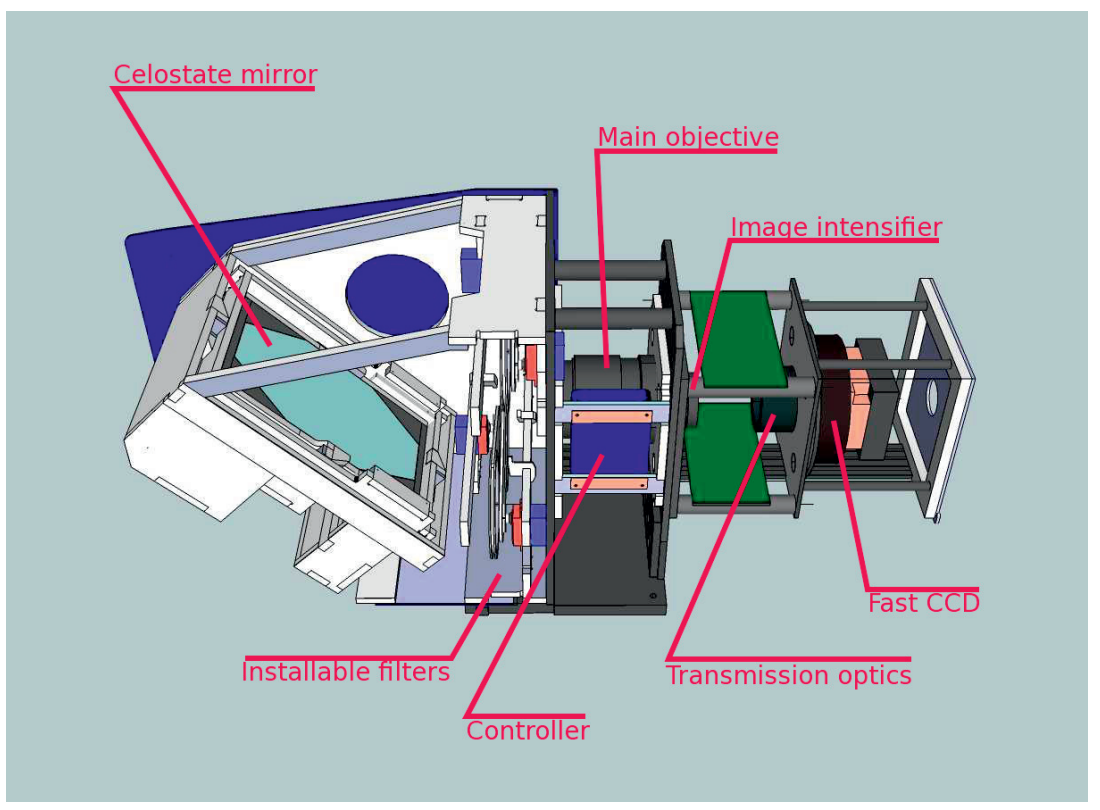

Fig. 1. Schematics of a single channel mechanical design of a MMT-Spain variant.

sensitivity of image intensifier microchannel plates, which drives it very important to perform a proper flat-fielding - and each channel therefore is equipped with its own flat-fielding module consisting of a dull surface on the inner part of a lid and dedicated photodiodes.

The mechanical scheme of a channel for this variant is shown in Figure 1.

MMT-Spain will be installed at El-Arenosillo atmospheric station in Huelva, Spain in fall 2013.

Second variant, MMT-Kazan, is equipped with Andor Neo sCMOS, which has $2560 \times 21606.4 \mu \mathrm{m}$ pixels with 16-bit depth. Due to limitations of a PC processing power, as well as available harddrives space, we decided to operate it in a 10 frames per second regime, which still provides us with $\sim 3 \mathrm{~Tb}$ of data per night. Quantum efficiency is about $55 \%$ with read-out noise as low as $1 \mathrm{e}^{-}$. Pixel scale is about $15^{\prime \prime}$ per pixel, and the channel field of view is about 100 square degrees. The limiting magnitude of a channel will be about $B \sim 12.5^{\mathrm{m}}$ in $0.1 \mathrm{~s}$, in both differential and direct imaging.

MMT-Kazan will be installed at Engelgardt observatory of Kazan Federal University, Kazan, Russia in summer 2013.

Both variants of MMT will use custom fork mounts based on a Skywatcher EQ6 head, each carrying two channels simultaneously. 
INTAS (04-78-7366), by the Presidium of the Russian Academy of Sciences Program, by the grant of President of Russian Federation for the support of young Russian scientists and by the grant of European Union (FP7 grant agreement number 283783, GLORIA project). The construction of MMT-Kazan is being financed by Kazan Federal University. S.K. has also been supported by a grant of Dynasty foundation. G.B. thanks Landau Network-Centro Volta and Cariplo Foundation for fellowship and Brera Observatory for hospitality. We thank Emilio Molinari, Stefano Covino and Cristiano Guidorzi for technical help organizing TORTORA observations and for discussions of the results.

\section{References}

Beskin, G., Bad'in, V., Biryukov, A., et al., 2005, Nuovo Cimento C, 28, 751

Beskin, G., Bondar, S., Karpov, S., et al., 2010a, Adv. Astron., 2010

Beskin, G., de-Bur, V., Karpov, S., Plokhotnichenko, V., \& Bondar, S., 2007, Bull. Spec. Astrophysical Observatory, 60-61, 217

Beskin, G., Karpov, S., Bondar, S., et al., 2010b, ApJ, 719, L10

Bondi, H., 1970, Q.J.R. Astron. Soc., 11, 443

Karpov, S., Bad'in, D., Beskin, G., et al., 2004, Astron. Nachr., 325, 677

Karpov, S., Beskin, G., Biryukov, A., et al., 2005, Nuovo Cimento C, 28, 747

Molinari, E., Bondar, S., Karpov, S., et al., 2006, Nuovo Cimento B, 121, 1525

Racusin, J.L., Gehrels, N., Holland, S.T., et al., 2008, GRB Coordinates Network Circular, 7427,1

Schaefer, B., 1985, AJ, 11, 1363

Schaefer, B., 1987, A\&A, 174, 338

Zolotukhin, I., Beskin, G., Biryukov, A., et al., 2004, Astron. Nachr., 325, 675 
